\title{
A 4-week, randomized, double-masked study to evaluate efficacy of deproteinized calf blood extract eye drops versus sodium hyaluronate $0.3 \%$ eye drops in dry eye patients with ocular pain
}

\author{
Yaying Wu", Xiuming Jin", Yujie Mou, Kelan Yuan, Jinjin Min, Xiaodan Huang \\ Eye Center, 2nd Affiliated Hospital, School of Medicine, Zhejiang University, Hangzhou, China \\ Contributions: (I) Conception and design: X Huang; (II) Administrative support: X Jin; (III) Provision of study materials or patients: X Huang, X Jin; (IV) \\ Collection and assembly of data: Y Wu, Y Mou, K Yuan, J Min; (V) Data analysis and interpretation: Y Wu; (VI) Manuscript writing: All authors; (VII) \\ Final approval of manuscript: All authors. \\ \#These authors contributed equally to this work. \\ Correspondence to: Xiaodan Huang, MD, PhD. Eye Center, Affiliated Second Hospital, School of Medicine, Zhejiang University, 88 Jiefang Road, \\ Hangzhou 310009, China. Email: huangxiaodan@zju.edu.cn.
}

\begin{abstract}
Background To evaluate the clinical efficiency of the treatment of dry eye disease (DED) with ocular pain using deproteinized calf blood extract (DCBE) eye drops as compared to $0.3 \%$ sodium hyaluronate (SH) eye drops.

Methods: This prospective, single-center, masked (double-blind), randomized controlled study included 53 patients divided into two groups: DCBE $(n=22)$ and $\mathrm{SH}(\mathrm{n}=31)$ group. The DCBE group received DCBE eye drops for 4 weeks, and the SH group received $0.3 \% \mathrm{SH}$ eye drops for 4 weeks. Corneal fluorescein staining (CFS) scores, tear break up time (TBUT), Schirmer test and the ocular surface disease index (OSDI) scores were evaluated in all patients before treatment, 2 and 4 weeks post-treatment.

Results: The DCBE group showed better improvement in the OSDI light sensitivity scores and ocular pain scores compared with the SH group $(\mathrm{P}<0.05)$. At 2 and 4 weeks post-treatment, the DCBE group and the SH group showed significant improvement in TBUT, Schirmer test, CFS, OSDI score, light sensitivity score and ocular pain score $(\mathrm{P}<0.05)$ compared with the data from before treatment.
\end{abstract}

Conclusions: This study indicates that DCBE eye drops can relieve ocular pain and light sensitivity in dry eye patients better than $\mathrm{SH}$ eye drops.

Keywords: Clinical trial; dry eye disease (DED); deproteinized calf blood extract eye drops; ocular pain

Submitted Jul 21, 2020. Accepted for publication Dec 23, 2020.

doi: 10.21037/apm-20-1453

View this article at: http://dx.doi.org/10.21037/apm-20-1453

\section{Introduction}

Major international epidemiological studies have concluded that the prevalence of dry eye disease (DED) ranges from $5 \%$ to $30 \%$ in individuals over the age of 50 (1). In DED, tear film instability and hyperosmolarity lead to inflammation, nociceptor neuron sensitivity, and peripheral nerve damage, which results in discomfort and pain. Eye pain in DED, including unpleasant dry eye sensations such as sore-irritated, gritty-scratchy, sharp, cutting, needlelike, pounding, or aching, may consist of nociceptive pain and neuropathic pain. DED-induced ocular pain and photophobia have serious effects in patients, and that discomfort will affect patients' quality of life to different degrees, such as being unable to read or drive a car, or even lead to depression (2). The objective of the TFOS DEWS II Pain and Sensation Subcommittee was to highlight the neurobiological mechanisms that underpin discomfort accompanying DED (3). It is important to find treatment for DED patients with adverse sensations of the ocular surface. 
The main treatment for DED is the partial supplement of artificial tears and auxiliary anti-inflammatory drugs in order to relieve patients' eye discomfort symptoms if necessary. However, clinical outcomes seem to indicate that artificial tears and anti-inflammatories are not enough for DED patients with ocular surface pain and sensitivity $(3,4)$. Newly developed medical concepts and technologies highlight that peripheral sensitization and neuroinflammatory responses participate in the development and maintenance of dry eye-related pain (5). Comfortable medical treatment has become a hot topic in recent years, which can accelerate the repair of the ocular surface and relieve dry eye symptoms. Researchers have observed that autologous serum tears (AST) could be used to treat photoallodynia in patients to reduce allodynia and hyperalgesia because AST is a rich source of growth factors that might improve the corneal epithelium and provide nutrition and energy for nerve regeneration $(6,7)$. It might be reasonable to link AST with deproteinized calf blood extract (DCBE). DCBE is a protein-free hemodialysate containing a broad spectrum of low molecular components of cellular mass and blood serum obtained from veal calves, and DCBE eye drops, a preparation with strong proepithelial repair effects, have been widely used in a variety of ocular surface diseases in recent years, showing good effects (8-14). It is worth studying whether the application of DCBE eye drops is effective in reducing discomfort and hyperesthesia, such as ocular pain and light sensitivity, in patients with mild or moderate DED, discussing their similarities and differences with the various types of pain experienced in other human pathologies.

We present the following article in accordance with the CONSORT reporting checklist (available at http://dx.doi. org/10.21037/apm-20-1453).

\section{Methods}

\section{Subjects}

This prospective study was conducted at the outpatient clinic of the Second Affiliated Hospital of Zhejiang University School of Medicine between August and November in 2019. This study followed the tenets of the Declaration of Helsinki (as revised in 2013) and was approved by the Ethics Committee of the Second Affiliated Hospital of Zhejiang University School of Medicine. Written informed consent was obtained from each participant after the nature and possible results of the study were explained (Trial registration number: ChiCTR1900022911).

The consecutive patients $\geq 18$ years of age with subjective dry eye-related hyperesthesia symptoms (light sensitivity or ocular pain), and tear break up time (TBUT) scores below 10 were recruited. The exclusion criteria included eye allergies, conjunctival inflammation, corneal ulcer, eyelid inflammation, severe blepharitis, palsy, valgus and other ocular lesions, severe systemic disease, and history of wearing contact lenses in the preceding 30 days.

Patients were randomly divided into the DCBE group and the SH group according to the random number table method. The DCBE group used DCBE eye drops (Qixing Pharmaceutical, Shenyang, China) 4 times a day for 4 weeks, while the SH group used $0.3 \%$ sodium hyaluronate eye drops (Santen Pharmaceutical, Osaka, Japan) with the same dosage, and both groups used fluorometholone eye drops (Santen Pharmaceutical) 4 times a day in the first 2 weeks. All patients were followed up at 2 and 4 weeks and after starting treatment.

Randomization was performed using opaque envelopes containing eye drops bottles. Both groups received these bottles and the examiner was masked to the group assignment. A consort flow diagram is presented in Figure 1.

\section{Dry eye symptom questionnaire}

Ocular surface disease index (OSDI) contains 12 different questions, and a $0-4$ score, from slight to severe degree, is given to each question. After aggregating the scores, the total score is $0-100$. An OSDI score over 13 was diagnosed as symptomatic DED. The OSDI score was evaluated again after 2 and 4 weeks of treatment.

\section{DED examination}

\section{Corneal fluorescence staining (CFS) score}

The cornea was divided equally into four quadrants, and the scoring was recorded after staining as follows: [0] no dye point; [1] 1-30 points; [2] more than 30 dye spots but no fusion; [3] entirely stained with fusion. A total score in each eye ranges from 0 to 12 .

\section{TBUT test}

Tear film instability was assessed using TBUT measurements. First, fluorescein was added to the tear film, and patients were asked to blink fully several times to ensure it was uniformly distributed across the ocular surface. The 


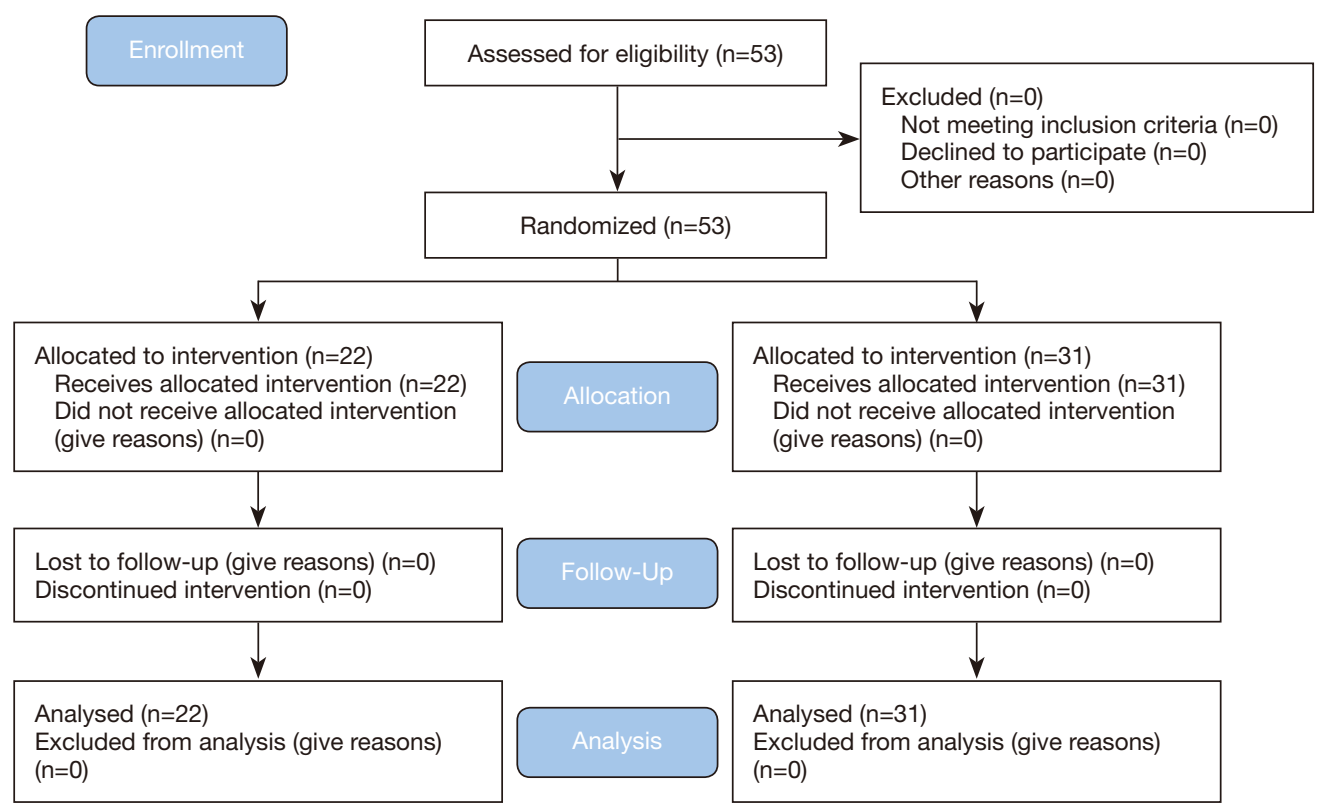

Figure 1 CONSORT 2010 flow diagram.

time between the last blink and the first dry spot on the tear film was measured.

\section{Tear secretion test}

After the application of a topical anesthesia, a filter paper strip $(35 \mathrm{~mm} \times 5 \mathrm{~mm})$ was put into the lower eyelid conjunctival sac, and the lengths of the wetted parts were recorded after 5 minutes. Lengths shorter than $10 \mathrm{~mm}$ were considered abnormal.

\section{Statistics}

All statistical analyses were performed using SPSS software (version 19, IBM, Armonk, NY, United States). Based on a previous study (11) sample size calculation, the study aimed to recruit patients in order to have a clinically significant difference with a confidence interval of $95 \%$ power $(\alpha=0.05)$. Data are presented as mean and standard deviation (SD) or as percentages. Mann-Whitney U-test is used for continuous or non-parametric data. And differences were assessed by using chi square or Fisher exact test for categorical variables. The Kolmogorov-Smirnov test and Spearman's rank-order correlation were used to identify the correlation between the TBUT, OSDI, CFS, and Schirmer test. An independent sample $t$-test was used to compare the parameters of the SH and DCBC groups. A P value $<0.05$ was considered statistically significant.

\section{Results}

This study included 53 Chinese patients with moderate dry eyes. In the DCBE group, there were 31 patients (11 men and 20 women; mean age $35.74 \pm 0.08$, range, $21-56$ years old), and in the $\mathrm{SH}$ group, there were 22 patients ( 8 men and 14 women; mean age $36.86 \pm 7.75$, range, $25-56$ years old). Before treatment, there was no statistical difference between the $\mathrm{SH}$ and DCBE groups $(\mathrm{P}>0.05)$ in CFS score, TBUT, Schirmer test, OSDI questionnaire scores, or even in the light sensitivity or ocular pain score. The baseline characteristics of the two groups and the results of the study are shown in Tables 1,2.

\section{DCBE eye drops can relieve OSDI symptoms in dry eye patients}

The DCBE group showed more improvement in the OSDI questionnaire scores than the $\mathrm{SH}$ group 4 weeks after treatment $(14.98 \pm 7.58$ vs. $22.41 \pm 6.11, \mathrm{P}<0.05)$, but not 2 weeks after treatment $(25.74 \pm 12.30$ vs. $25.37 \pm 7.23$, $\mathrm{P}>0.05)$. Compared with before treatment, the OSDI score improved significantly after 2 weeks of treatment in the DCBE group $(\mathrm{P}<0.05)$, and a significant difference was also found after 4 weeks of treatment $(\mathrm{P}<0.05)$. The significant differences in the DCBE group were found in the $\mathrm{SH}$ group as well $(\mathrm{P}<0.05)$ (Figure 2). 
Table 1 Baseline data for SH group and DCBE group

\begin{tabular}{lcc}
\hline Variables & SH & DCBE \\
\hline Patients $(\mathrm{n})$ & 22 & 31 \\
Age (years) & $36.86 \pm 7.75$ & $35.74 \pm 10.08$ \\
Men, $\mathrm{n}(\%)$ & $8(36.4)$ & $11(35.5)$ \\
Women, $\mathrm{n}(\%)$ & $14(63.6)$ & $20(64.5)$ \\
TBUT & $5.56 \pm 2.32$ & $4.90 \pm 2.26$ \\
CFS score & $2.98 \pm 1.51$ & $2.85 \pm 1.43$ \\
Tear secretion test & $5.80 \pm 1.67$ & $5.77 \pm 1.51$ \\
OSDI & $38.24 \pm 10.77$ & $42.55 \pm 14.97$ \\
Light sensitivity & $3.00 \pm 0.78$ & $2.96 \pm 0.71$ \\
Ocular pain & $3.00 \pm 0.67$ & $3.00 \pm 0.79$ \\
\hline
\end{tabular}

Data are expressed as the mean \pm standard deviation. $\mathrm{SH}$, sodium hyaluronate eye drops; DCBE, deproteinized calf blood extract eye drops; TBUT, tear break-up time; CFS, corneal fluorescein staining; OSDI, ocular surface disease index.

Table 2 Comparison of indicators before and after treatment

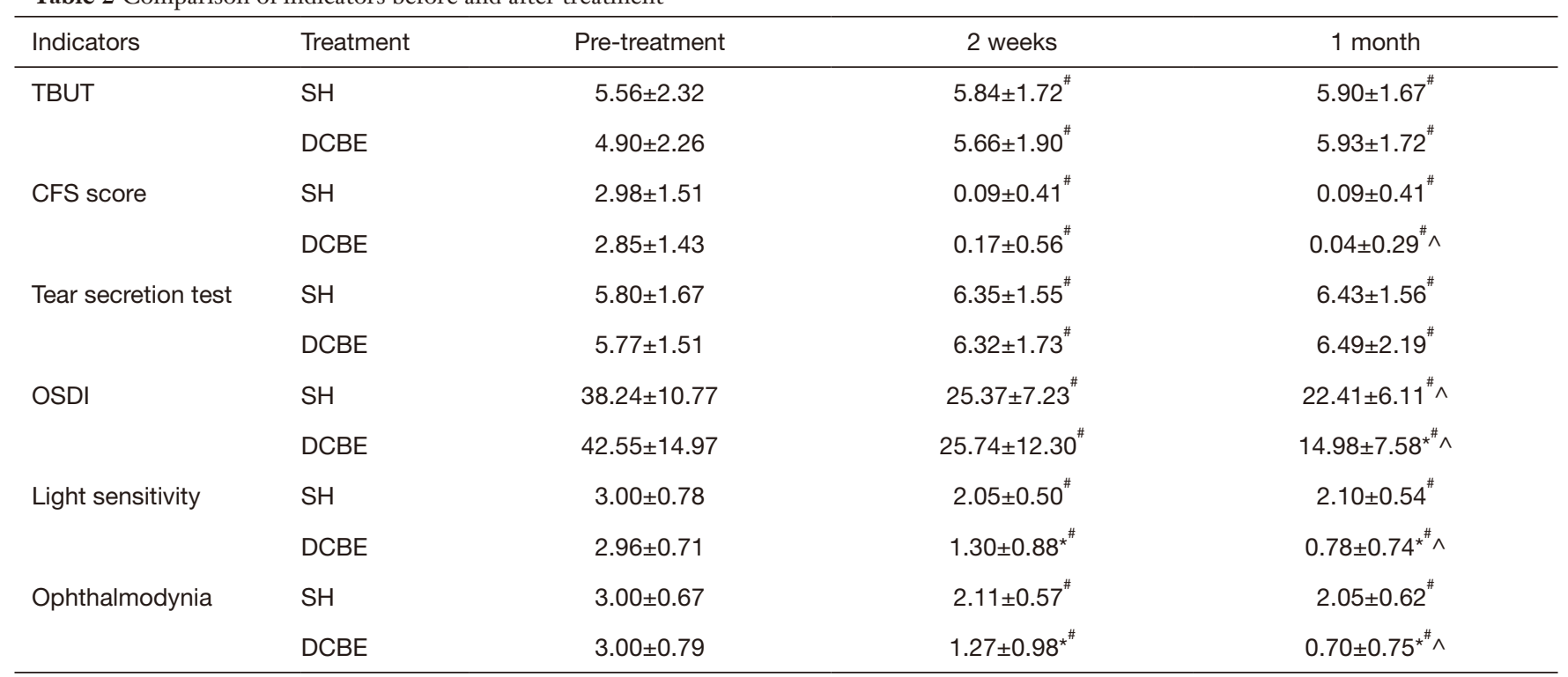

Data are expressed as the mean \pm standard deviation. * , statistically significant DCBE compared to $\mathrm{SH}$ group, $\mathrm{P}<0.05$; " , statistically significant compared to pre-treatment, $\mathrm{P}<0.05 ; \wedge$, statistically significant 1 -month treatment compared to 2 -week treatment, $\mathrm{P}<0.05$. TBUT, tear break-up time; CFS, corneal fluorescein staining; OSDI, ocular surface disease index; SH, sodium hyaluronate eye drops;

DCBE, deproteinized calf blood extract eye drops.

\section{DCBE eye drops can relieve dry eye-related hyperesthesia symptoms}

There were 23 patients in the DCBE group and 21 patients in the $\mathrm{SH}$ group who suffered from obvious light sensitivity (light sensitivity score $\geq 2$ in OSDI questionnaire scores), and 31 patients in the DCBE group and 19 patients in the SH group suffered from serious ocular pain (ocular pain score $\geq 2$ in OSDI questionnaire scores). Before treatment, there was no statistical difference between these two groups (DCBE group vs. SH group in light sensitivity $2.96 \pm 0.71 v s$. 


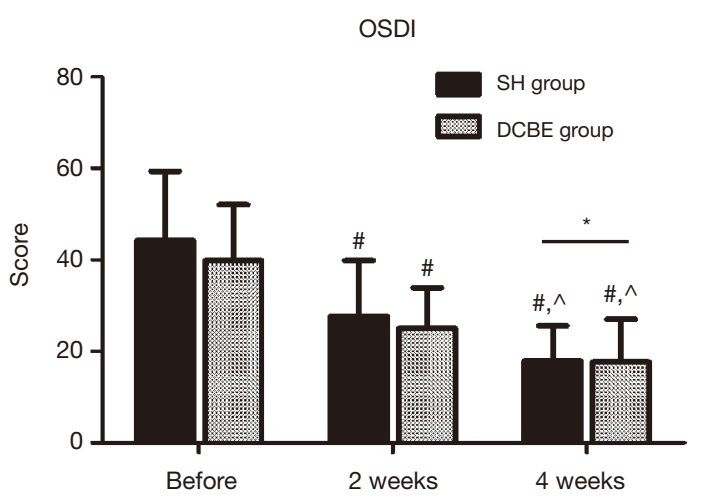

Figure 2 OSDI scores in DCBE and SH groups 2 and 4 weeks after treatment. *, statistically significant compared to $\mathrm{SH}$ group, $\mathrm{P}<0.05$; \#, statistically significant compared to pre-treatment, $\mathrm{P}<0.05$; ^, statistically significant compared to 2-week treatment, $\mathrm{P}<0.05$. OSDI, ocular surface disease index; $\mathrm{SH}$, sodium hyaluronate eye drops; DCBE, deproteinized calf blood extract eye drops.

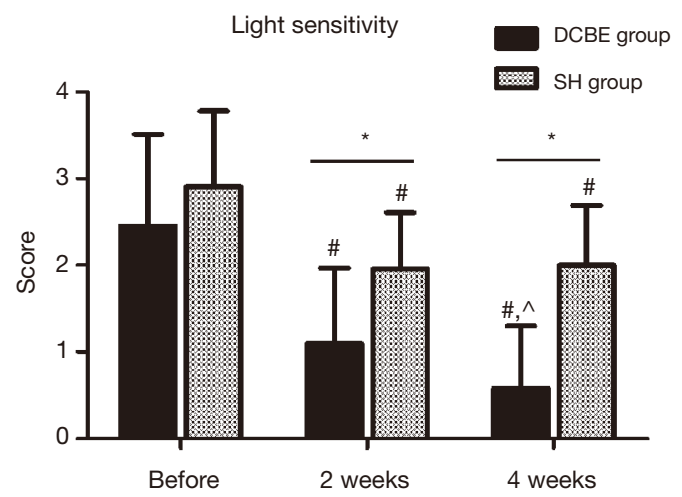

Figure 3 Light sensitivity scores in DCBE and SH groups 2 and 4 weeks after treatment. *, statistically significant compared to $\mathrm{SH}$ group, $\mathrm{P}<0.05$; ", statistically significant compared to pretreatment, $\mathrm{P}<0.05 ; \wedge$, statistically significant compared to 2 weektreatment, $\mathrm{P}<0.05$. $\mathrm{SH}$, sodium hyaluronate eye drops; DCBE, deproteinized calf blood extract eye drops.

$3.00 \pm 0.78$; in ocular pain $3.00 \pm 0.79$ vs. $3.00 \pm 0.67, \mathrm{P}>0.05$ ). After 2 weeks of treatment, the DCBE group improved more in ocular pain scores $(2.11 \pm 0.57$ vs. $1.27 \pm 0.98)$ and light sensitivity scores $(1.30 \pm 0.88$ vs. $2.05 \pm 0.50)$ than the $\mathrm{SH}$ group. After 4 weeks of treatment, the differences in the DCBE and SH groups were more significant in ocular pain score $(0.70 \pm 0.75$ vs. $2.05 \pm 0.62, \mathrm{P}<0.05)$ and in light sensitivity score $(0.78 \pm 0.74$ vs. $2.10 \pm 0.54, \mathrm{P}<0.05)$. In the

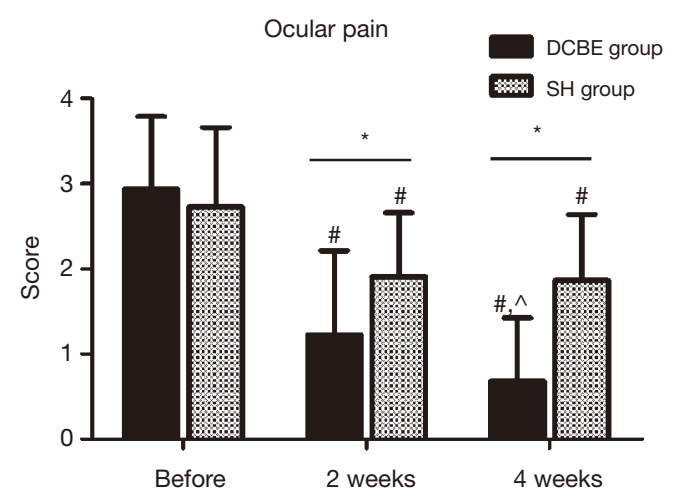

Figure 4 Ocular pain scores in DCBE and SH groups 2 and 4 weeks after treatment. *, statistically significant compared to SH group, $\mathrm{P}<0.05$; \#, statistically significant compared to pretreatment, $\mathrm{P}<0.05 ;{ }^{\wedge}$, statistically significant compared to 2 -week treatment, $\mathrm{P}<0.05$. $\mathrm{SH}$, sodium hyaluronate eye drops; $\mathrm{DCBE}$, deproteinized calf blood extract eye drops.

DCBE group, there were significant differences at 4 weeks post-treatment compared with 2 weeks post-treatment $(\mathrm{P}<0.05)$. In the $\mathrm{SH}$ group, there were no significant differences between 4 weeks and 2 weeks post-treatment $(\mathrm{P}>0.05)$ in the ocular pain and light sensitivity scores (Figures 3,4).

\section{DCBE eye drops can improve CFS}

There was no statistical difference between the DCBE group and the $\mathrm{SH}$ group in the CFS score 2 weeks after treatment $(0.17 \pm 0.56$ vs. $0.09 \pm 0.41, \mathrm{P}>0.05)$. No statistical difference was found after 4 weeks between the groups $(0.04 \pm 0.29$ vs. $0.09 \pm 0.41, \mathrm{P}>0.05)$. The downtrend in score dropped quickly in the first 2 weeks of treatment, and there were statistical differences in groups separately $(\mathrm{P}<0.05)$ but no statistical difference in the second 2 weeks in each group $(\mathrm{P}>0.05)$.

\section{TBUT after treatment}

There was no significant difference between the DCBE group and the $\mathrm{SH}$ group after the first 2 weeks of treatment $(5.66 \pm 1.90$ vs. $5.84 \pm 1.72, \mathrm{P}>0.05)$ as well as after 4 weeks of treatment $(5.93 \pm 1.72$ vs. $5.90 \pm 1.67, \mathrm{P}>0.05)$. In the DCBE group, statistical differences could be found in the first 2 weeks of treatment as well as in the second 2 weeks $(\mathrm{P}<0.05)$, 
while in the SH group, compared with before treatment, we found a significant difference after 4 weeks of treatment $(\mathrm{P}<0.05)$ but not in the first 2 weeks or in the second 2 weeks $(\mathrm{P}>0.05)$.

\section{Tear secretion test after treatment}

The was no significant difference between the DCBE and $\mathrm{SH}$ groups after 2 weeks of treatment $(6.32 \pm 1.73 \mathrm{vs}$. $6.35 \pm 1.55 \mathrm{~mm}, \mathrm{P}>0.05)$ or after 4 weeks $(6.49 \pm 2.19$ vs. $6.43 \pm 1.56 \mathrm{~mm}, \mathrm{P}>0.05)$. Both groups improved in the tear secretion test in the first 2 weeks $(\mathrm{P}<0.05)$ but not in the second 2 weeks $(\mathrm{P}>0.05)$.

No obvious side effects occurred during the follow-up, and no abnormal intraocular pressure was detected.

\section{Discussion}

DED is generally believed to be largely caused by a loss of homeostasis of the tear film, which will leave the corneal epithelium exposed to hyperosmolarity and ocular surface inflammation. Hyperosmolarity and inflammation lead to acute nociceptive pain and thermoreceptor sensitizing. Prolonged stimulating may result in peripheral nerve damage and neuropathic pain, which may disrupt neuroimmune communication in DED. Due to inflammatory processes, corneal nerve injury followed by altered excitability in regenerated nerves may result in the development of hyperalgesia or allodynia in patients with DED $(15,16)$. When pain persists past the normal time of healing, it becomes chronic or persistent pain (15-17). The objective of the TFOS DEWS II Pain and Sensation Subcommittee was to highlight the neurobiological mechanisms that underpin discomfort accompanying DED (3). It is important to find treatment for DED patients with adverse sensations of the ocular surface.

Although ophthalmologists now pay more attention to the sensitivity of patients with DED, and more researchers have focused on the unpleasant sensory and emotional experiences of DED, the treatment options are still limited $(4,15,18)$. In the literature, artificial tears (19-22) and antiinflammatory medications are solutions for reducing nerve sensitivity, but sometimes, they are not very effective $(23,24)$. A subset of dry eye patients has ocular surface pain, and these patients are likely to be more resistant to topical therapy directed at optimizing the ocular surface $(4,17)$. As mentioned above, nociceptive and neuropathic pain are not mutually exclusive and can coexist in ocular surface pain
$(4,23,25,26)$. The relationship between corneal sensitivity and disease severity is often confused because the onset time of DED is not clear. In some recent studies, AST was used in patients with corneal neuropathy-induced photoallodynia and severe corneal pain and showed profound alterations in corneal nerves $(27,28)$. AST restores nerve topography through nerve regeneration, and this was correlated with improvement in patient-reported sensitivity, but there are limitations on the feasibility of ocular surface pain neuroregenerative therapy, namely, that AST is difficult to use widely in China due to its high preparation cost and preservation requirements. DCBE eye drops mainly contain a variety of free amino acids, low molecular peptides, and oligosaccharides, containing a broad spectrum of low molecular components of cellular mass and blood serum obtained from veal calves. This combination improves the transport of oxygen and glucose to cells under hypoxic conditions. It increases the synthesis of intracellular ATP and contributes to an increase in the level of aerobic glycolysis and oxidative phosphorylation. It activates reconditioning and regeneration in tissues by stimulating fibroblast proliferation and collagen vascular wall repair. DCBE provides nutrients and energy for promoting tissue repair and cell regeneration of nerves. DCBE has been widely used in a variety of ocular surface diseases in recent years, showing good improvement in patients suffering from ocular surface symptoms, such as corneal epithelial defects, or in patients with xerophthalmia after LASIK, LASEK, or pterygium surgery, with filamentary keratitis, or with severe dry eye induced by chronic graft-versus-host disease after bone marrow transplantation (8-14).

In our study, both the DCBE and SH groups showed improvement in objective signs and subjective symptoms. Particularly in the DCBE group, ocular pain and light sensitivity continued improving, and the curative effect was more significant. This might suggest that DCBE has an indication in DED patients with ocular pain and light sensitivity. Inflammation plays an important role in the pathogenesis and chronicity of DED, and so fluorometholone eye drops were used to reduce the risks of inflammation in our first 2 weeks of treatment. We suggest that in the first 2 weeks, fluorometholone eye drops reduced inflammation and improved ocular surface scores in some ways in both groups. As shown in the previous research of Benowitz et al., Bukowiecki et al. and Pflugfelder et al., inflammation represents a major driving force in sensitization, and anti-inflammation plays an important role in the regeneration of the peripheral sensory neurons 
(29-31). Our results also demonstrated that the DCBE group experienced an improved curative effect and better improvement in OSDI and ocular pain and light sensitivity scores in the third and fourth weeks of treatment even without fluorometholone eye drops, which may suggest that DCBE has similar effects to AST in corneal epithelium and nerve healing.

Our study has some limitations. First, we still need a larger number of participants. In the future, there will be a larger-population, long-term, multi-center study for further research. It was found that central sensitization in non-ocular pain conditions is frequently associated with the status of depression and anxiety, which suggests that an assessment of depression and anxiety level might be necessary (32-34), The identification of the neurological mechanisms underlying pain is important in order to define therapies. We can use a corneal in vivo confocal microscope (IVCM) to observe corneal neuropathy and subepithelial plexus changes and to monitor disease severity and therapeutic effects. A corneal IVCM allows high-resolution in vivo visualization of sub-basal corneal nerves and immune cells at a cellular level, which can be monitored for disease severity and response to treatment, as shown in the studies of Aggarwal et al. (27,28). With tests of tear cytokines and neuropeptides, we will find a better explanation of the mechanism in the treatment and management of DED (3).

\section{Conclusions}

This study indicated that DCBE eye drops can improve both ocular pain and light sensitivity in dry eye patients. DCBE eye drops may be more effective than artificial tears for dry eye patients with ocular pain.

\section{Acknowledgments}

Funding: This work was supported by the National Natural Science Foundation of China (grant No. 81870624; 81700802); Major Science and Technology Projects of Zhejiang Province (grant No. 2017C03046).

\section{Footnote}

Reporting Checklist: The authors have completed the CONSORT reporting checklist. Available at http://dx.doi. org/10.21037/apm-20-1453

Data Sharing Statement: Available at http://dx.doi. org/10.21037/apm-20-1453

Conflicts of Interest: All authors have completed the ICMJE uniform disclosure form (available at http://dx.doi. org/10.21037/apm-20-1453). The authors have no conflicts of interest to declare.

Ethical Statement: The authors are accountable for all aspects of the work in ensuring that questions related to the accuracy or integrity of any part of the work are appropriately investigated and resolved. This study followed the tenets of the Declaration of Helsinki (as revised in 2013) and was approved by the Ethics Committee of the Second Affiliated Hospital of Zhejiang University School of Medicine. Written informed consent was obtained from each participant after the nature and possible results of the study were explained (Trial registration number: ChiCTR1900022911).

Open Access Statement: This is an Open Access article distributed in accordance with the Creative Commons Attribution-NonCommercial-NoDerivs 4.0 International License (CC BY-NC-ND 4.0), which permits the noncommercial replication and distribution of the article with the strict proviso that no changes or edits are made and the original work is properly cited (including links to both the formal publication through the relevant DOI and the license). See: https://creativecommons.org/licenses/by-nc-nd/4.0/.

\section{References}

1. The epidemiology of dry eye disease: report of the Epidemiology Subcommittee of the International Dry Eye WorkShop (2007). Ocul Surf 2007;5:93-107.

2. Smart KM, Blake C, Staines A, et al. Self-reported pain severity, quality of life, disability, anxiety and depression in patients classified with 'nociceptive', 'peripheral neuropathic' and 'central sensitisation' pain. The discriminant validity of mechanisms-based classifications of low back (+/-leg) pain. Man Ther 2012;17:119-25.

3. Belmonte C, Nichols JJ, Cox SM, et al. TFOS DEWS II pain and sensation report. Ocul Surf 2017;15:404-37.

4. Kojima T, Dogru M, Kawashima M, et al. Advances in the diagnosis and treatment of dry eye. Prog Retin Eye Res 2020. [Epub ahead of print]. doi: 10.1016/ j.preteyeres.2020.100842.

5. Fakih D, Zhao Z, Nicolle P, et al. Chronic dry eye induced corneal hypersensitivity, neuroinflammatory responses, 
and synaptic plasticity in the mouse trigeminal brainstem. J Neuroinflammation 2019;16:268.

6. Bernabei F, Roda M, Buzzi M, et al. Blood-Based Treatments for Severe Dry Eye Disease: The Need of a Consensus. J Clin Med 2019;8:1478.

7. Petznick A, Tong L, Chung R, et al. Autologous plasma eyedrops prepared in a closed system: a treatment for dry eye. Eye (Lond) 2013;27:1102.

8. Deng S, Wang M, Zhang F, et al. Corneal subbasal nerve fiber regeneration in myopic patients after laser in situ keratomileusis. Neural Regen Res 2012;7:1556-62.

9. Qiu XD, Gong L, Sun XH, et al. Efficacy of Protein-Free Calf Blood Extract for Mechanical Corneal Epithelial Defects in Human Eyes. Zhonghua Yan Ke Za Zhi 2008;44:720-5.

10. Chen MJ, Gong L, Qiu XD. Efficacy of Protein-Free Calf Blood Extract Ophthalmic Gel for Diffuse Punctate Corneal Epithelial Defects. Zhonghua Yan Ke Za Zhi 2012;48:1083-7.

11. Niu LL, Zhou XT, Ding L, et al. The Effects of ProteinFree Calf Blood Extract for Recovery of Corneal Nerve After LASEK and LASIK. Zhonghua Yan Ke Za Zhi 2011;47:539-45.

12. Lin ZR, Wu HP, Xie ZW, et al. Efficacy of Deproteinized Calf Blood Extract Eye Drops on Early Recovery After Pterygium Surgery. Zhonghua Yan Ke Za Zhi 2019;55:134-40.

13. Lv H, Liu Z, Li X, et al. Effect of lacrimal plugs combined with deproteinized calf blood extract eye gel for filamentary keratitis. J Ocul Biol Dis Infor 2010;3:134-40.

14. Liu J, Liu ZG, Shao Y, et al. The clinical efficiency of calf blood extract gel on moderate to severe dry eye induced by chronic graft versus host diseases after bone marrow transplantation. Zhonghua Yan Ke Za Zhi 2013;49:32-6.

15. Siedlecki AN, Smith SD, Siedlecki AR, et al. Ocular pain response to treatment in dry eye patients. Ocul Surf 2020;18:305-11.

16. Moshirfar M, Benstead EE, Sorrentino PM, et al. Ocular Neuropathic Pain. In: StatPearls. Treasure Island (FL): StatPearls Publishing, 2020.

17. Bron AJ, de Paiva CS, Chauhan SK, et al. TFOS DEWS II pathophysiology report. Ocul Surf 2017;15:438-510. Erratum in: Ocul Surf. 2019 Oct;17(4):842. doi: 10.1016/ j.jtos.2019.08.007. Epub 2019 Aug 8.

18. Jones L, Downie LE, Korb D, et al. TFOS DEWS II Management and Therapy Report. Ocul Surf 2017;15:575-628.
19. Yang G, Espandar L, Mamalis N, et al. A crosslinked hyaluronan gel accelerates healing of corneal epithelial abrasion and alkali burn injuries in rabbits. Vet Ophthalmol 2010;13:144-50.

20. Ho WT, Chiang TH, Chang SW, et al. Enhanced corneal wound healing with hyaluronic acid and high-potassium artificial tears. Clin Exp Optom 2013;96:536-41.

21. Pinto-Fraga J, López-de la Rosa A, Blázquez Arauzo F, et al. Efficacy and Safety of $0.2 \%$ Hyaluronic Acid in the Management of Dry Eye Disease. Eye Contact Lens 2017;43:57-63.

22. Park Y, Song JS, Choi CY, et al. A randomized multicenter Study comparing $0.1 \%, 0.15 \%$, and $0.3 \%$ sodium hyaluronate with $0.05 \%$ cyclosporine in the treatment of dry eye. J Ocul Pharmacol Ther 2017;33:66-72.

23. Dermer H, Lent-Schochet D, Theotoka D, et al. A Review of Management Strategies for Nociceptive and Neuropathic Ocular Surface Pain. Drugs 2020;80:547-71.

24. Goyal S, Hamrah P. Understanding Neuropathic Corneal Pain-Gaps and Current Therapeutic Approaches. Semin Ophthalmol 2016;31:59-70.

25. Basbaum AI, Bautista DM, Scherrer G, et al. Cellular and molecular mechanisms of pain. Cell 2009;139:267-84.

26. Galor A, Levitt RC, Felix ER, et al. Sarantopoulos CD. Neuropathic ocular pain: an important yet underevaluated feature of dry eye. Eye (Lond) 2015;29:301-12.

27. Aggarwal S, Kheirkhah A, Cavalcanti BM, et al. Autologous Serum Tears for Treatment of Photoallodynia in Patients with Corneal Neuropathy: Efficacy and Evaluation with In Vivo Confocal Microscopy. Ocul Surf 2015;13:250-62.

28. Aggarwal S, Colon CM, Kheirkhah A, et al. Efficacy of Autologous Serum Tears for Treatment of Severe Corneal Pain in Patients with Corneal Neuropathy: An In Vivo Confocal Microscopy Study. Invest Ophthalmol Vis Sci 2014;55:1468.

29. Bukowiecki A, Hos D, Cursiefen C, et al. Wound-Healing Studies in Cornea and Skin: Parallels, Differences and Opportunities. Int J Mol Sci 2017;18:1257.

30. Benowitz LI, Popovich PG. Inflammation and axon regeneration. Curr Opin Neurol 2011;24:577-83.

31. Pflugfelder SC. Antiinflammatory therapy for dry eye. Am J Ophthalmol 2004;137:337-42.

32. Labbé A, Wang YX, Jie Y, et al. Dry eye disease, dry eye symptoms and depression: the Beijing Eye Study. Br J Ophthalmol 2013;97:1399-403. 
33. McNally L, Bhagwagar Z, Hannestad J. Inflammation, glutamate, and glia in depression: a literature review. CNS Spectr 2008;13:501-10.

Cite this article as: $\mathrm{Wu} \mathrm{Y,} \mathrm{Jin} \mathrm{X,} \mathrm{Mou} \mathrm{Y,} \mathrm{Yuan} \mathrm{K,} \mathrm{Min} \mathrm{J,}$ Huang X. A 4-week, randomized, double-masked study to evaluate efficacy of deproteinized calf blood extract eye drops versus sodium hyaluronate $0.3 \%$ eye drops in dry eye patients with ocular pain. Ann Palliat Med 2021;10(4):3617-3625. doi: 10.21037/apm-20-1453
34. Wen $\mathrm{W}, \mathrm{Wu} \mathrm{Y}$, Chen $\mathrm{Y}$, et al. Dry eye disease in patients with depressive and anxiety disorders in Shanghai. Cornea 2012;31:686-92. 